\title{
Gait modification for improving walking stability of exoskeleton assisted paraplegic patient
}

\author{
Mengze Li ${ }^{*} \mathbb{D}$, Tadayoshi Aoyama and Yasuhisa Hasegawa
}

\begin{abstract}
The currently available exoskeleton for assisting the paraplegic patient in walking usually adapts a pre-programmed gait that involves the patient following an exoskeleton lead. The system allows the patient to hold a pair of canes in order to keep balance, and does not contribute to keeping balance without the patient's action. This paper proposes an algorithm based on the zero moment point (ZMP) to modify the gait generated through human walking synergy for paraplegic patients who make use of the exoskeleton system and hold their canes. The proposed ZMP will enable the paraplegic patient to keep balance during walking and also reduce the burden in maintaining balance. First, a pair of cane is used as an interface to control the user's walking and then, the synergy between legs and canes is used to synchronize the user's walking intention during the exoskeleton movement. The walking synergy is extracted from the able-bodied subject walking with a pair of canes and analyzed using principal component analysis (PCA). In order to improve the walking stability, the hip joint angle on stance leg during walking was modified based on ZMP. Furthermore, a nonlinear inverted pendulum (NIP) model was utilized in order to generate a gait with a fully stretched knee joint angle that is similar to human gait. The proposed method was verified via the Gazebo simulation using a walking robot to simulate a patient wearing an exoskeleton. The experiment results show that the walking stability was highly improved after gait modification.
\end{abstract}

Keywords: Human walking synergy, Lower-limb exoskeleton, Walking stability

\section{Introduction}

In recent years, research on the powered exoskeleton has become a hot topic. The rapid development of the exoskeleton has made great achievements in two aspects: (1) augmentation in human strength and durability. For example: Berkeley lower extremity exoskeleton is designed to increase human endurance and strength [1]. Quasi-passive leg utilizes only spring and damper to augment load-carrying during walking [2]. (2) restoration in the physical function of a disabled patient. For example: Roboknee determines user intent through the knee joint angle and ground reaction forces and allows the wearer to climb stairs [3]. Re-walk enables spinal cord injured

\footnotetext{
*Correspondence: li@robo.mein.nagoya-u.ac.jp

Department of Micro-Nano Mechanical Science and Engineering, Nagoya University, 1 Furo-cho, Chikusa-ku, Nagoya 464-8603, Japan
}

patients to walk without human assistance [4]. NeuroRex uses non-invasive electroencephalography to decode a paraplegic patient motion intent and aid walking [5]. LOPES applied impedance control on joints to allow bidirectional mechanical interaction between robot and patient for gait rehabilitation [6].

Among the above-mentioned exoskeletons, exoskeletons for assisting the paraplegic patient have been widely used in scientific research, rehabilitation, and daily life. A paraplegic patient is a person who has lost the motor and sensory function of the lower body. The exoskeleton acts directly on the patient's body, supporting the user's weight and augment the strength as well as provides a high capability for the paraplegic patient to walk again. Sankai et al. [7] developed a well-known exoskeleton, hybrid assistive limb (HAL), to help physically challenged people to walk again. HAL uses electromyography to 
estimate the walking intention to support a paraplegic patient to walk. In [8], HAL detects a preliminary motion to enhance the transfer between standing and sitting for complete paraplegic patients. In [9], HAL infers the spinal cord injury patient's walking intention using the ground reaction force for gait support. In [10], HAL estimates the leg swing speed according to the walking velocity for restoring the gait of spinal cord injury patients. Besides, Ekso Bionics developed by Ekso measures the position of the user's center of gravity and estimates the walking intention of paraplegic patients by detecting the center of mass (CoM) transfer when their upper body is leaned forward [11].

All the above-mentioned exoskeletons adapt a pre-programmed joint trajectory for walk assistance. The user holds a pair of canes to keep balance which supports the exoskeleton's movements to walk. Although the start and stop of walking is controlled by the user incline the body, the user still needs to adjust the angle and fall point of the canes to cooperate with the gait of the exoskeleton. The pre-programmed trajectory method is not the only solution to generate the exoskeleton movement. The researches about human walking synergy make non-preprogrammed trajectory generation possible.

Researches on human walking synergy have reported that human walking does not only involve a repetitive swing of legs but a highly coupled cooperative motion between upper and lower limbs. Matthew et al. examined the synergy between arms and legs in healthy adults by constraining one arm while walking in a treadmill [12]. Thierry et al. proved that the synergy between arms and legs exist not only during human walking but also in creeping and swing activities [13]. Jaclyn et al. studied the synergy between the arms and legs during cycling movement and stated that "any contributions from the arms is functionally linked to locomotion" [14]. Volker et al. studied the synergy between arms and legs by measuring the electromyographic on leg and arms during walking [15]. Volker also studied the synergy between the arms and legs of patients suffering from movement disorders [16]. Besides, Principal component analysis (PCA) is often used to analyze human synergy to extract the coupling relationship between limbs. Daffertshofer et al. published a tutorial about how to apply PCA on moment data as feature extractor and as data-driven filter [17]. Todorov er al. analyzed the synergies underlying complex hand manipulation using PCA [18].

The human synergy also greatly contributed to the design and control of exoskeleton. Crocher et al. proposed a robot control approach that integrates an explicit model of inter-joint coordination based on a linear relationship between joint velocities $[19,20]$. Liu developed a rehabilitation exoskeleton based on the postural synergy that allows the 10 degrees of freedom robot driven by only two actuators [21]. Hassan proposed an instrumented cane to help hemiplegic patients walk with the help of an exoskeleton [22-24]. Hemiplegic patients are patients who lost the motor and sensory function of half side of the body but another side remains functional. In their studies, the motion of the unaffected leg and cane were used to induce the motion of the affected leg. In our study, the target patient is paraplegic. Paraplegic patients are patients who lost the motor and sensory function of the lower body but the upper body remains functional. We researched the synergy between canes (arms) and legs and confirmed that by using the motion of canes will aid to generate the motion of legs for walking.

This study uses a cane as an interface to control the walk of a paraplegic patient wearing an exoskeleton. The walking synergy was extracted from a healthy subject and applied to synchronize the exoskeleton's movement with the user's intention. To improve the walking stability, the hip joint trajectory on the stance leg was modified using the zero moment point (ZMP). To generate a human-like gait, a non-linear inverted pendulum (NIP) model was used to modify the walking trajectory generated by walking synergy. Therefore, our approach does not give additional operations to paraplegic patients. The proposed gait generation and modification method were validated by simulation using the Gazebo software. The major contribution in this paper is the gait modification method based on ZMP to improve the walking stability of a paraplegic patient wearing an exoskeleton. Using this system, the exoskeleton also contributes to the balance control, thus reduce the burden of keeping the balance of the patient while walking. The minor contribution of this paper is on the utilization of canes as an interface to synchronize the user's intention and leg motion. It is noteworthy to mention that using the cane as the interface does not increase the burden of the patients as the purpose is only to keep balance.

This paper is organized as follows: "Gait generation based on walking synergy" section introduces PCA to extract the walking synergy between cane and leg motion from data of subject walking with canes. "Motion planning using NIP and ZMP" section introduces ZMP and NIP for motion planning. "Gait modification using NIP and ZMP" section involves steps on how to combine the human walking synergy and ZMP based gait modification. "Simulation and result" section introduces the walking robot and verifies the proposed gait modification method in the Gazebo simulation. "Discussion" section discusses the advantage and possible improvement of the proposed gait modification method. Finally, the concluding remarks is presented at the end of the paper. 


\section{Gait generation based on walking synergy}

The detail explanation of using principal component analysis (PCA) to analyze human walking synergy is described in $[25,26]$. PCA combines the variables to derive new components and as a result produce a simpler description of the system. The matrix of the principal components (eigenvectors in descending order) is divided and rearranged to calculate the unknown variables from the known variables.

$$
y=\Gamma^{T} x
$$

where $\Gamma$ is the matrix of the eigenvectors in descending order concerning the eigenvalues. The original data $x$ is mapped to the new data $y$ after being multiplied by the liner transition matrix $\Gamma^{T}$. Since the $\Gamma$ is an orthonormal matrix, then $x$ could be rewritten as:

$$
x=\Gamma y .
$$

then $\Gamma$ could be separated for the known variables $x_{1}$ and the unknown variables $x_{2}$ :

$$
x_{1}=\Gamma_{1} y, \quad x_{2}=\Gamma_{2} y .
$$

where $\Gamma_{1}$ and $\Gamma_{2}$ are the separated matrix for the known and unknown variables, respectively. The unknown variables could be computed from the separated matrixes and the known variables:

$$
x_{2}=\Gamma_{2} \Gamma_{1}^{\sharp} x_{1} .
$$

where $\Gamma_{1}^{\sharp}$ is the pseudo-inverse of $\Gamma_{1}$ :

$$
\Gamma_{1}^{\sharp}=\left(\Gamma_{1}^{T} \Gamma_{1}\right)^{-1} \Gamma_{1}^{T} .
$$

In the case of a paraplegic patient, the matrix $x$ is the data of joint angle from a subject walking with a cane, the known variable matrix $x_{1}$ is the joint angle of the upper limbs, and the unknown variable matrix $x_{2}$ is the joint trajectories of the lower limbs. Instead of applying the arm joint trajectories, the cane inclination were used as the known variable matrix $x_{1}$. In the case of this paper, the extracted synergy is contained in a 6 by 6 matrix, and each column corresponds to a principal component (PC). Each $\mathrm{PC}$ has a contribution ratio to the synergy, and we show the contribution ratio in Table 1 . We chose PC1, PC2, and PC3 as the matrix $\Gamma$, where the first two rows are $\Gamma_{1}$ and the last four rows are $\Gamma_{2}$. In this way, the walking synergy was used as a gait generator, with the cane inclination angle as input, and the generator generates a joint angle of hip and knee. Therefore the $x_{1}$ and $x_{2}$ can be written as:

$$
\begin{aligned}
& x_{1}=\left[\begin{array}{ll}
\theta_{l} & \theta_{r}
\end{array}\right]^{T}, \\
& x_{2}=\left[\begin{array}{llll}
\theta_{s w}^{h} & \theta_{s w}^{k} & \theta_{s p}^{h} & \theta_{s p}^{k}
\end{array}\right]^{T} .
\end{aligned}
$$

where $\theta_{l}$ and $\theta_{r}$ are the left and right cane inclination angles. The $\theta_{s w}^{h}$ and $\theta_{s w}^{k}$ are the hip and knee joint angle of the swing leg, the $\theta_{s p}^{h}$ and $\theta_{s p}^{k}$ are the hip and knee joint angle of the support leg. The $\theta_{s p}^{k}$ will be replaced to improve the walking stability, and the method is introduced in following section.

Three subjects participated in this experiment and comprise of all-male, healthy, aged from 25 to 30 and without any history of movement disorder. The gait of the paraplegic patient wearing the exoskeleton was related to the degree of injury and the proficiency of exoskeleton used. A trained paraplegic patient with both unaffected arms can swing a cane and leg on the opposite side simultaneously during walking. The utilized gait is the fastest and the most efficient, therefore it becomes the target motion in this research and is used to extract the walking synergy. To mimic the walking conditions of the paraplegic patient, the subjects were asked to support the body with crutches as much as possible during the walk while moving the cane with the leg on the opposite side simultaneously during walking.

The body kinematics was recorded using a 3D optical motion capture system (OptiTrack) at a frequency of 250 Hz. 16 infrared reactive markers were fixed on the lower body of the subject to record the walking movement and 3 markers on each cane to record the movement of canes. The 16 infrared reactive markers were fixed according to the conventional lower limb model, 2 on each foot, 1 on each ankle, 1 on each shank, 1 on each the knee, 1 on each thigh, and 4 on the waist.

Figure 1 shows the comparison between the joint angle of the human walk and the joint angle generated using walking synergy. For the hip joint angle, the mean error and the maximum errors are 1.51 [deg] and 5.47 [deg], respectively. In the case of the knee joint angle, the mean error and maximum errors are 2.54 [deg] and 15.96 [deg], respectively. The small discrepancy shows that the generated joint angle has high similarity with the joint of human walking.

\section{Motion planning using NIP and ZMP}

The ZMP is widely used for gait generation in a humanoid robot. For example, Philippe strictly defined the ZMP in [27], and Kajita used a preview control of ZMP for biped gait generation [28]. Linear inverted pendulum (LIP) model is widely used in the trajectory planning $[29,30]$. The LIP assumes a constant height for the center of mass (CoM) which results in a bent knee joint on the stance leg during gait. During the stance phase of the human walking the 

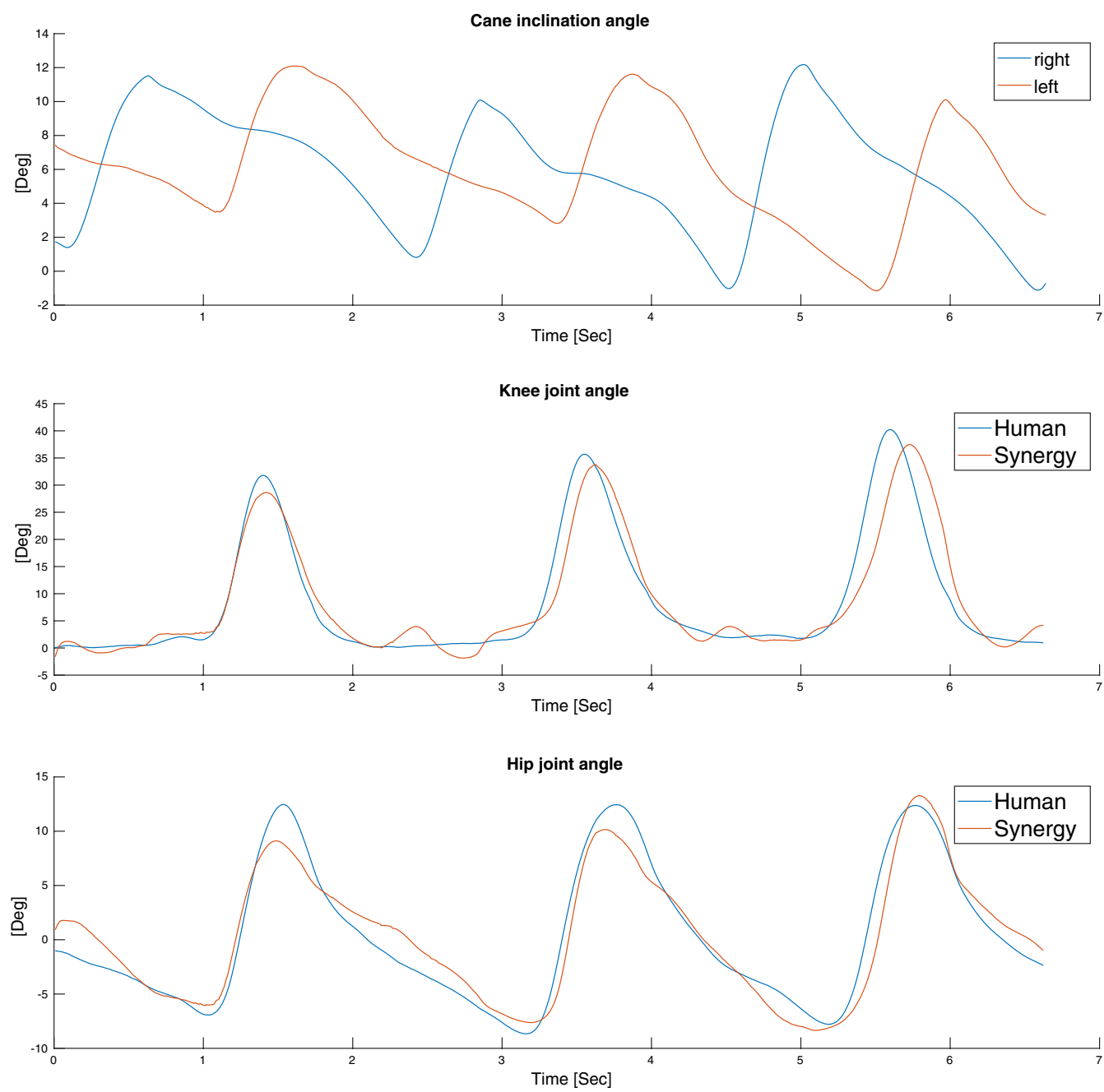

Fig. 1 Comparison between human walking joint trajectory and walking joint angles generated using walking synergy

stance leg becomes fully extended, which makes the LIP model not in accordance with the natural human walking. In the present study we use a nonlinear inverted pendulum (NIP) to generate the CoM trajectory, subsequently, the leg joint angle can be obtained by solving the inverse kinematics. For the NIP, the length of the pendulum remains constant and the generated walking motion has a fully stretched knee joint on stance leg, which is similar to the human walking habit.

Figure 2 shows the nonlinear inverted pendulum (NIP) model. The inverted pendulum model has a constant length $r$, the CoM is assumed concentrated at the tip of the pendulum, and the angle between the pendulum and the vertical direction is $\theta$. The position of the CoM can be written as follow:

$$
x_{M}=r \sin (\theta), z_{M}=r \cos (\theta) .
$$

The zero moment point $x_{Z M P}$ can be derived from this model:

$$
\tau=m\left(g+\ddot{z}_{M}\right)\left(x_{M}-x_{Z M P}\right)+m \ddot{x}_{M} z_{M} .
$$

where $m$ is the mass at center, $g$ is the gravitational acceleration, $\tau$ is the total external moment, $x_{M}$ and $z_{M}$ are the CoM position, $\ddot{x}_{M}$ and $\ddot{z}_{M}$ is the acceleration in the direction along the coordinate $X$ and $Z$. The resultant moment $\tau$ at the zero moment point equals to zero can be written as:

$$
\ddot{x}_{M}=\frac{\ddot{z}_{M}+g}{z_{M}}\left(x_{M}-x_{Z M P}\right) .
$$

Due to the high degree of nonlinearity, the differential Eq. (9) is difficult to find an analytical solution. Assume the $Z_{M}$ is constant, this model becomes a linear inverted pendulum (LIP), and the solution can be written as: 


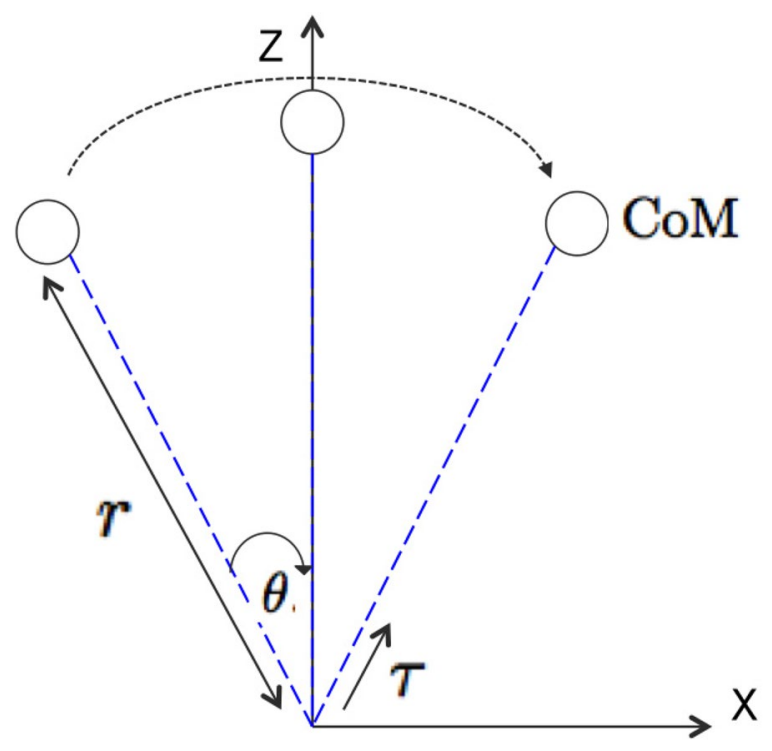

Fig. 2 Nonlinear inverted pendulum model

Table 1 Ratio of principal components

\begin{tabular}{llllll}
\hline PC1 (\%) & PC2 (\%) & PC3 (\%) & PC4 (\%) & PC5 (\%) & PC6 (\%) \\
\hline 50.71 & 37.74 & 7.28 & 2.83 & 1.44 & 0.01 \\
\hline
\end{tabular}

$$
\begin{aligned}
x_{M} & =C_{1} e^{\omega}+C_{2} e^{-\omega}+x_{Z M P}, \\
\omega & =t /\left(z_{M} / g\right)^{\frac{1}{2}} .
\end{aligned}
$$

where $z_{M}$ is a constant. Given the CoM position and walking cycle, as well as the reference ZMP, then the $C_{1}$ and $C_{2}$ can be determined, and the trajectory of CoM can be written as a function of time. From Eq. (7), we can obtain the below equation:

$$
\theta=\sin ^{-1}\left(\frac{x_{M}}{r}\right)
$$

By substituting the solution (10) into the Eqs. (11) and (7), we can get the trajectory of $\theta$ and $z_{M}$. Furthermore, by differentiating $z_{M}$ we can get $\ddot{z}_{M}$. From Eq. (9), we can get:

$$
x_{Z M P}=x_{M}-\frac{\ddot{x}_{M} z_{M}}{\ddot{z}_{M}+g} .
$$

By substituting $z_{M}$ and $\ddot{z}_{M}$ into the Eq. (12), we can get the trajectory of ZMP.

Figure 3 shows an example of CoM trajectory planning using a nonlinear inverted pendulum model. We calculated the difference between ZMP and reference ZMP for different pendulum lengths and step lengths. It was found that this difference is mainly related to the ratio between
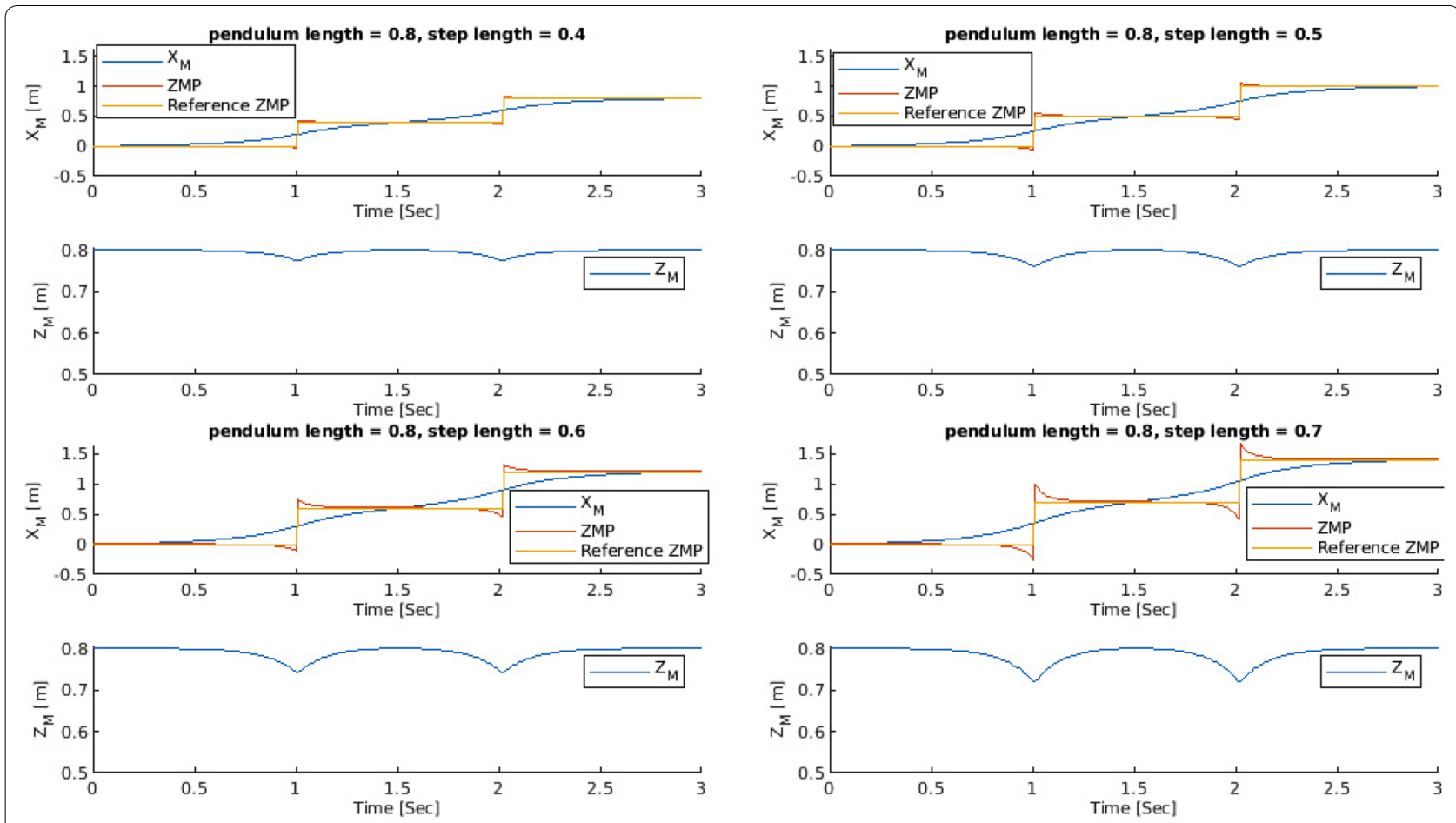

Fig. 3 Example of ZMP planning using a nonlinear inverted pendulum model 
the step length and pendulum length. When the ratio is less than 0.5, there is almost no difference between ZMP and reference ZMP. When the ratio is above 0.5 , an error is observed and the error increases with an increase in the ratio. In this case, it is necessary to judge whether the error has a significant effect on the stability of walking (i.e. whether the ZMP still falls within the supporting polygon). In this paper, the ratio is maintained at 0.5 or less.

\section{Gait modification using NIP and ZMP}

The walking gait generated from the walking synergy might not result in a fully stable walk due to the dynamic difference between the lower-limb exoskeleton and a human subject. Therefore, to make the walking synergybased gait fits with the patient wearing the powered exoskeleton, we modified the walking gait by employing ZMP.

The walking synergy is used as a gait generator, with the motion of the cane as input and the walking synergy generates the joint angle of legs for walking. In the real case, the IMU sensor can be attached to the cane to measure its inclination. The NIP model can be used to generate a stable CoM trajectory, in which the resultant ZMP stays in the support polygon formed by the cane and foot. The key point is to use the NIP to generate a stance leg motion that is in accordance with the swing leg motion generated by walking synergy. The hip joint on stance leg is modified to improve the walking stability. We considered two points when using the ZMP to modify the walking motion: (1) The generated walking motion should have a fully stretched knee joint on stance leg which fits the human walking habit. (2) There should be no gap at the joint angle when the swing leg becomes a stance leg. When a human walks, the knee joint of stance leg is fully stretched. If the length of the NIP model equals the length of the leg when it is fully stretched, the walking motion generated using the NIP has a fully stretched knee joint on the stance leg. Given the walking cycle and step length, the hip joint trajectory of the stance leg can be generated using NIP. In addition, given the joint angle when the swing leg becomes the stance leg, the hip joint trajectory can be modified so that there exists no gap on the joint angle trajectory when the swing leg becomes the stance leg. The details can be described below.

Figure 4 shows the relationship between the pendulum and human walking in gait modification. The origin of the pendulum is at the contact point between the pendulum and ground while the origin of the human is at the contact point between the stance leg and ground. Assuming the step length of walking is $l$, and the CoM of the pendulum moves from $-l / 2$ to $l / 2$, the moment of the human to start a step and the pendulum to move is at time $t_{s}$, and the moment of the human to end the step and when the pendulum stops moving is at time $t_{e}$. By substituting the above-mentioned values into Eq. (10), we can get:

$$
\begin{aligned}
-l / 2 & =C_{1} a_{1}+C_{2} a_{2}+x_{Z M P}, \\
l / 2 & =C_{1} a_{3}+C_{2} a_{4}+x_{Z M P} .
\end{aligned}
$$

where the $a_{1}, a_{2}, a_{3}$ and $a_{4}$ are:

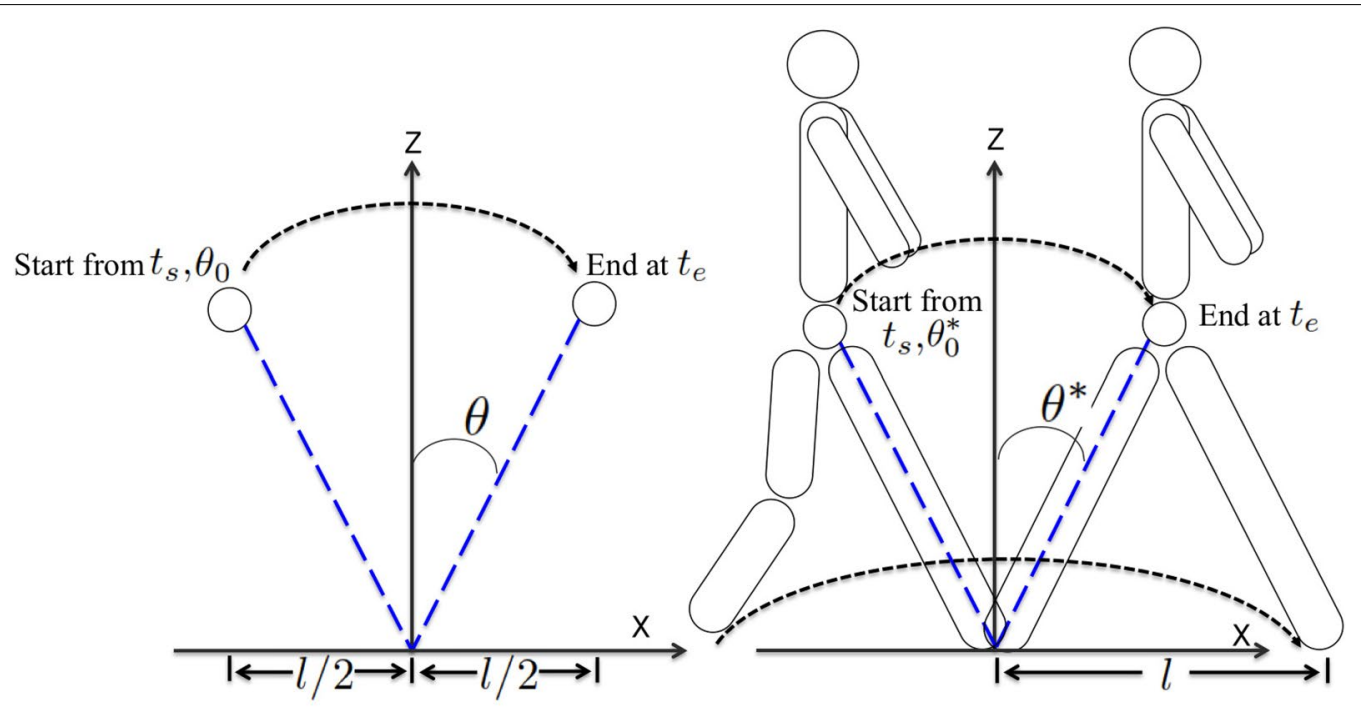

Fig. 4 Relationship between pendulum and human walking in gait modification 


$$
\begin{aligned}
& a_{1}=e^{-t_{s} /\left(z_{M} / g\right)^{\frac{1}{2}}}, \\
& a_{2}=e^{t_{s} /\left(z_{M} / g\right)^{\frac{1}{2}}}, \\
& a_{3}=e^{-t_{e} /\left(z_{M} / g\right)^{\frac{1}{2}}}, \\
& a_{4}=e^{t_{e} /\left(z_{M} / g\right)^{\frac{1}{2}}} .
\end{aligned}
$$

Therefore, the parameters to determine the solution function of Eq. (10) can be obtained as follow:

$$
\begin{aligned}
& C_{1}=\frac{a_{2} l / 2+a_{4} l / 2}{a_{1} a_{4}-a_{2} a_{3}}, \\
& C_{2}=\frac{a_{1} l / 2+a_{3} l / 2}{a_{1} a_{4}-a_{2} a_{3}} .
\end{aligned}
$$

Using Eq. (11), we can convert the solution of Eq. (10) into an inclination angle of the pendulum $\theta$. As shown in figure 4 , assuming the hip joint angle on stance leg when a step is taken is $\theta_{0}^{*}$, the inclination angle of the pendulum when it starts to move is $\theta_{0}$, the modified hip joint angle trajectory of stance $\theta^{*}$ can be obtained by:

$$
\theta^{*}=\theta-\left(\theta_{0}-\theta_{0}^{*}\right) .
$$

where $\theta^{*}$ is used to replace the hip joint angle of support leg, $\theta_{s p}^{h}$, which is mentioned in "Gait generation based on walkingsynergy" section .

Different from a fully programmed walking system, the time consumed and step length of each step are unknown in advance, and they may vary in each step. To use the ZMP to modify the hip joint trajectory on stance leg, the time consumed and the step length needs to be predicted in advance for each step.

Since walking is a highly coupled cooperative motion between upper and lower limbs, the walking cycle and step length can be related to the motion of cane. When the patient walks using a trot-like gait, the opposite leg and the cane starts and lands almost at the same time, and the relative distance between them is almost constant. In our method, the walking cycle and step length are predicted in each step using the angle change of the cane when the cane commenced moving. Walking cycle usually refers to the time consumed during walking and includes one left step and one right step. After prediction at the beginning of each step, half of the walking cycle was used as the time consumed to generate the walking motion using ZMP.

Figure 5 shows the relationship between the walking cycle and angular change of cane in 0.08 [s] as well as between step length and angular change of cane in 0.08 [s]. This Figure includes the data of 50 steps of three subjects walking with a cane. The $\mathrm{x}$-axis is the angle change of the cane in 0.08 [s] after it starts to swing whereas the

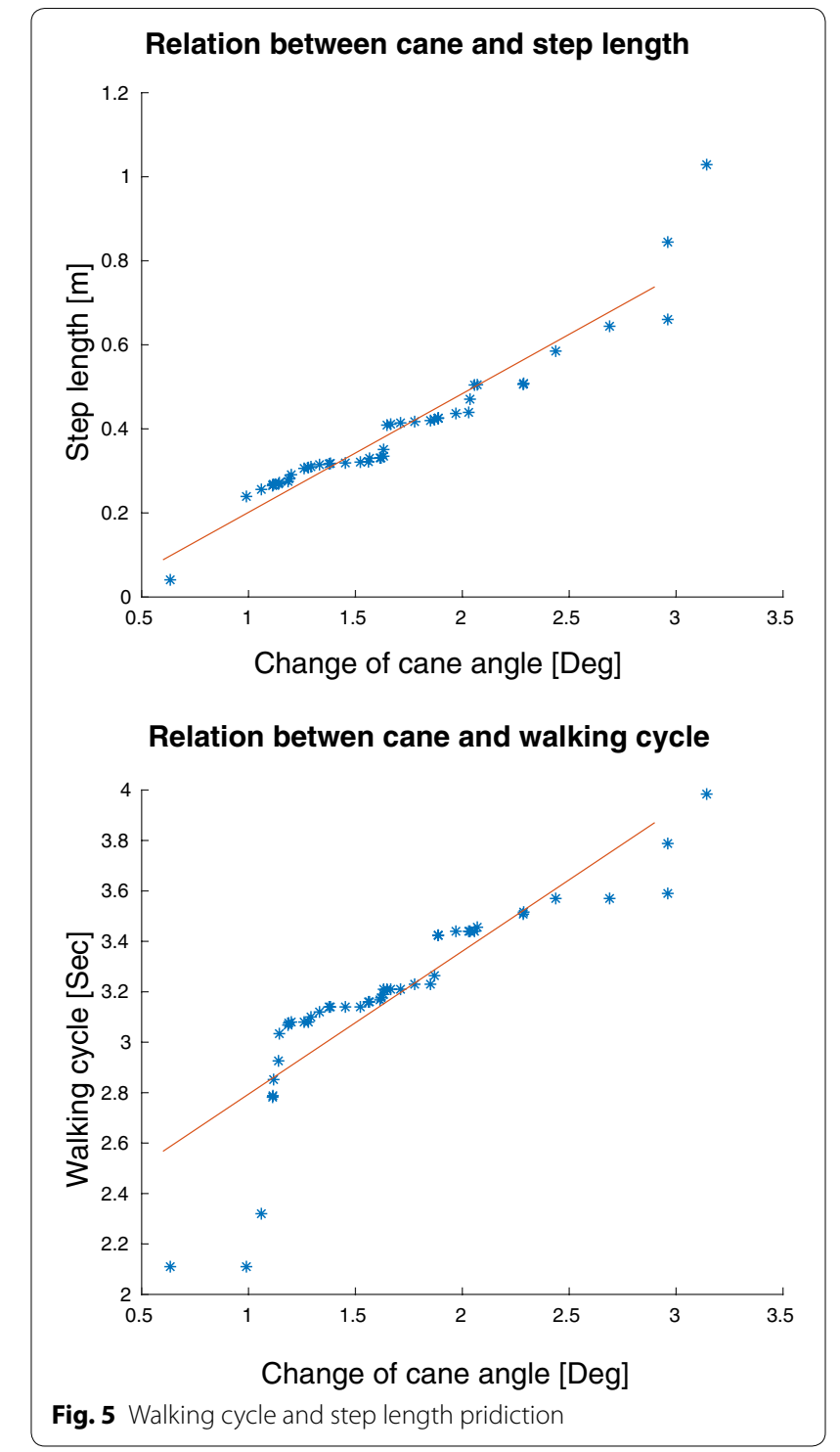

$y$-axis refers to the walking cycle and step length, respectively. The equation to predict the walking cycle and step length are obtained from this Figure and can be written as:

$$
\begin{aligned}
& T=0.567 A_{\text {cane }}+2.228 . \\
& S=0.283 A_{\text {cane }}-0.081 .
\end{aligned}
$$

where $T$ is the walking cycle and $S$ is the step length, and $A_{\text {cane }}$ is the angle change of the cane in $0.08[\mathrm{~s}]$ after it started to swing.

During walking, deviations between predictions and actual conditions may occur. The error of walking cycle prediction will cause the foot and the cane to land at different time. If the error is small, the stability of walking 


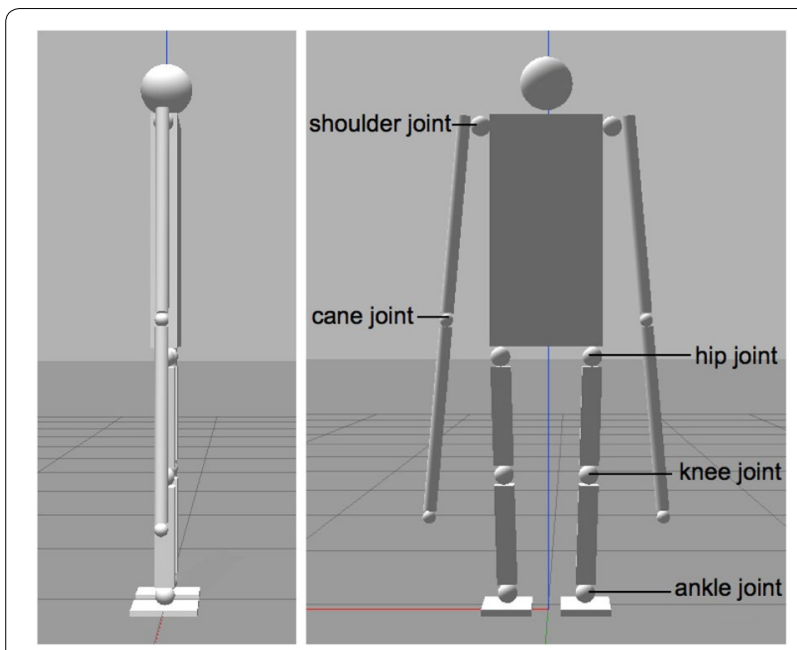

Fig. 6 Walking robot replacing a patient for preliminary study

Table 2 Parameters of walking robot

\begin{tabular}{lcclll}
\hline Parameter/Part & Thigh & Lower & Foot & Body & Crutch \\
\hline Mass $[\mathrm{kg}]$ & 1.742 & 1.774 & 0.592 & 12.48 & 2.24 \\
Length $[\mathrm{cm}]$ & 33.3 & 32.75 & 20 & 73 & 134 \\
\hline
\end{tabular}

becomes unaffected. However, the error of step length prediction accumulates as the walking distance increases and needs to be corrected via compensation. To avoid the accumulation of errors, the error of the last step was compensated at the previous steps:

$$
S_{K}^{\prime}=S_{K}+E_{K-1},
$$

where $S_{K}^{\prime}$ is the step length updated in the current step, $S_{K}$ is the step length predicted in the current step, $E_{K-1}$ is the error measured in the last step.

\section{Simulation and result}

It is dangerous to conduct experiments with a paraplegic patient before validating the feasibility of the proposed method. Furthermore, an able-bodied subject cannot wear an exoskeleton to replace the patient used for validating experiments. Although able-bodied subjects can consciously imitate paraplegic patients and can subconsciously put force into their lower limbs to keep their balance when the risk of falling is high. Therefore, a walking robot was built to simulate a paraplegic patient wearing an exoskeleton to conduct a preliminary study for safety reasons.

Figure 6 shows the schematic illustrations of the walking robot. The inertia and link lengths of the lower body are shown in Table 2 . The robot height is $180[\mathrm{~cm}]$ while the width is $70[\mathrm{~cm}]$. The weight of the walking robot is
25 [kg] with 16 degrees of freedom. The movable joints are the shoulder joints, hip joints, knee joints, and ankle joints. Each leg has six degrees of freedom, the hip has three degrees of freedom, the knee has one degree of freedom, the ankle has two degrees of freedom. The shoulder has one degree of freedom, and the crutches can be stretched and shortened meanwhile the robot can adjust its center of gravity to maintain balance. Each joint of the robot is equipped with an encoder that measures the joint angle during the experiments. To measure the ZMP during walking, a six-axis $\mathrm{F} / \mathrm{T}$ sensor was installed at the ankle joint.

We performed a robot walking experiment during the simulation to verify the feasibility of the proposed method. The walking was divided into the walking phase and stance phase. The walking phase is a single-support phase while the stance leg supports the body weight and swing leg in the air for step taking. The transfer of the CoM happens at the walking phase and the stance legs aid the CoM to move forward. The stance phase is a double-support phase and both legs support the body weight. This is a transitional phase, in which the robot remains still and does not transfer its CoM. In the simulation, the stance phase takes 1 [sec] and the time consumed at the walking phase is related to the cane motion and its walking cycle.

We compared the robot walking with and without gait modification. In the case of robot walking without gait modification, the motion of the cane was extracted from a subject walking with a cane, and the gait was generated by human walking synergy using the cane motion as input. In the case of the robot walking with gait modification (modified using ZMP), the motion of the cane was extracted from a subject walking with a cane, the gait was generated by human walking synergy using the cane motion as input and was modified using ZMP.

Figure 7 shows the simulation scene of robot walking with gait modification. The upper image in Fig. 7 is the front view of the robot walking and the lower image in Fig. 7 is the side view of the robot walking. In a real case scenario, the exoskeleton is an under-actuated system, it has degrees of freedom in the sagittal and lateral plane, but it only provides support in the sagittal plane. In the simulation, the balance in the lateral plane was kept by the cane.

Figure 8 shows the ZMP measurement results of the walking experiment. The upper image in Fig. 8 is the ZMP of robot walking with gait modification. The lower image in Fig. 8 is the ZMP of robot walking without gait modification. In the case of robot walking with gait modification, the maximum error between ZMP and reference ZMP was 0.204 [m] with an average error of $0.028[\mathrm{~m}]$. Errors mostly occur when the 

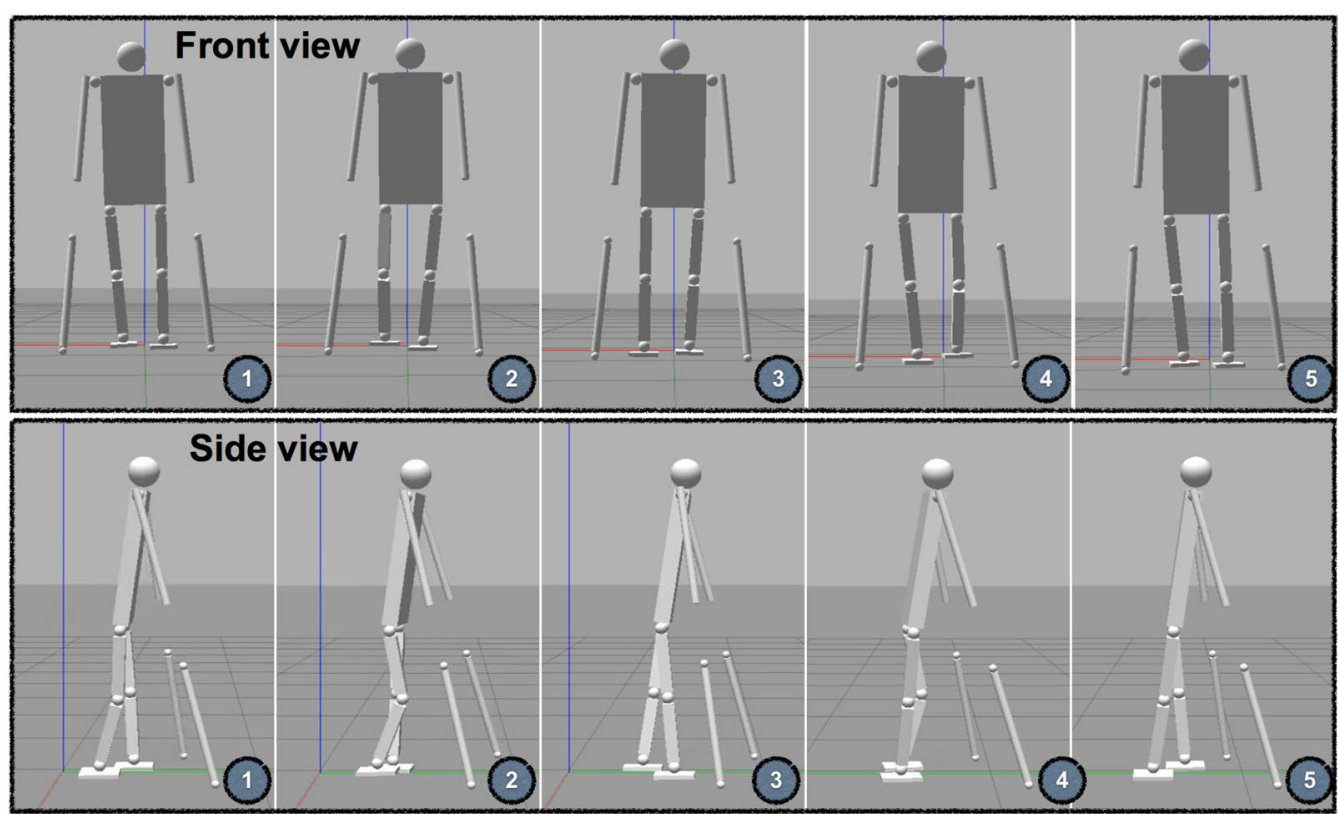

Fig. 7 Simulation scene of robot walking

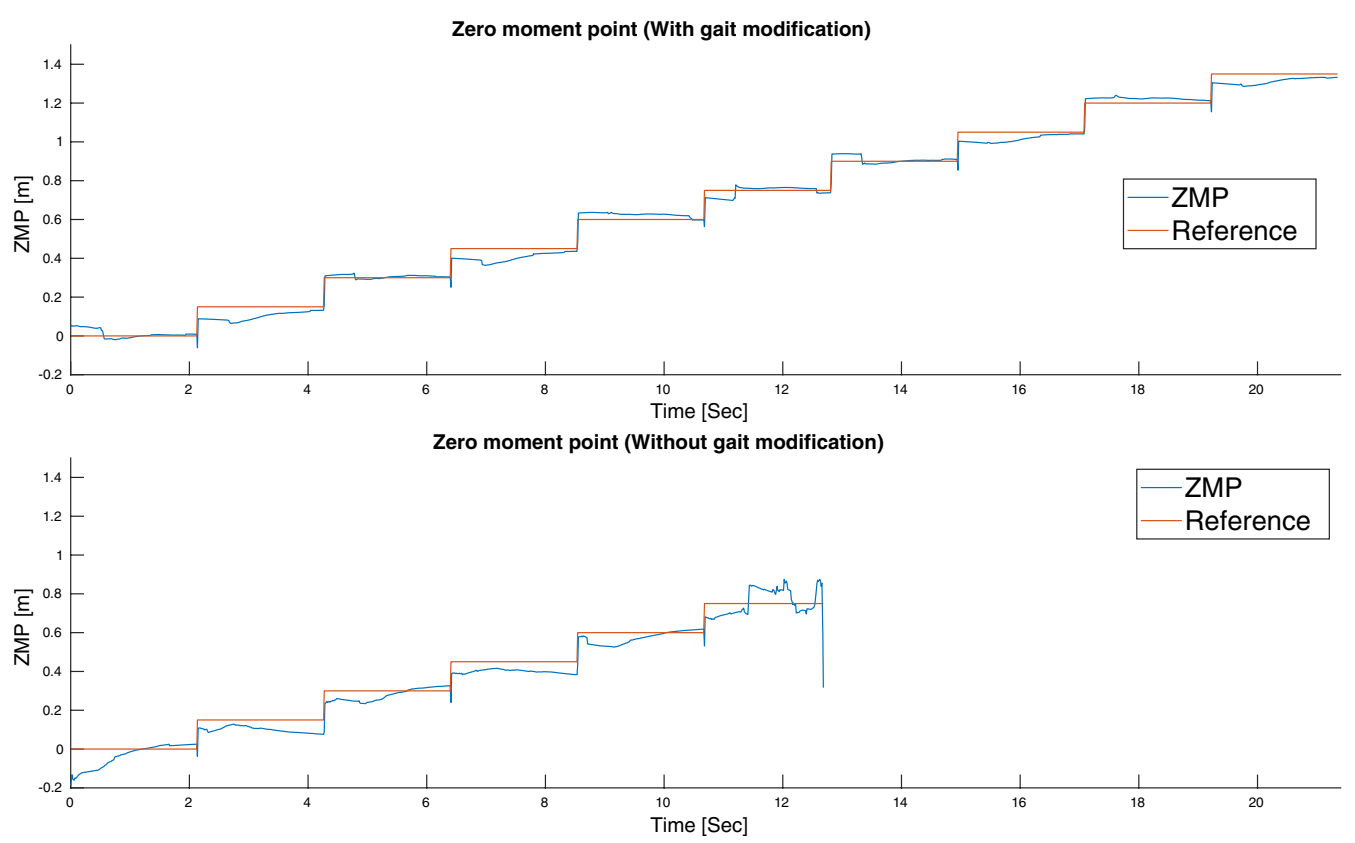

Fig. 8 Comparison between robot walking with and without gait modification

robot takes a step and the ZMP gradually converges to the reference ZMP. This is mainly due to the robot change from stationary to motion, and the maintenance of balance is changed from 4-point to 2-point support. Although there is a difference between the $\mathrm{ZMP}$ and the reference ZMP, the ZMP always stays in the support polygon while preventing the robot from falling. In the case of walking without gait modification, the maximum error between ZMP and reference ZMP was $0.434[\mathrm{~m}]$ with an average error of 0.050 $[\mathrm{m}]$. Errors mostly occur when the robot takes a step with no convergence of ZMP to the reference ZMP, 

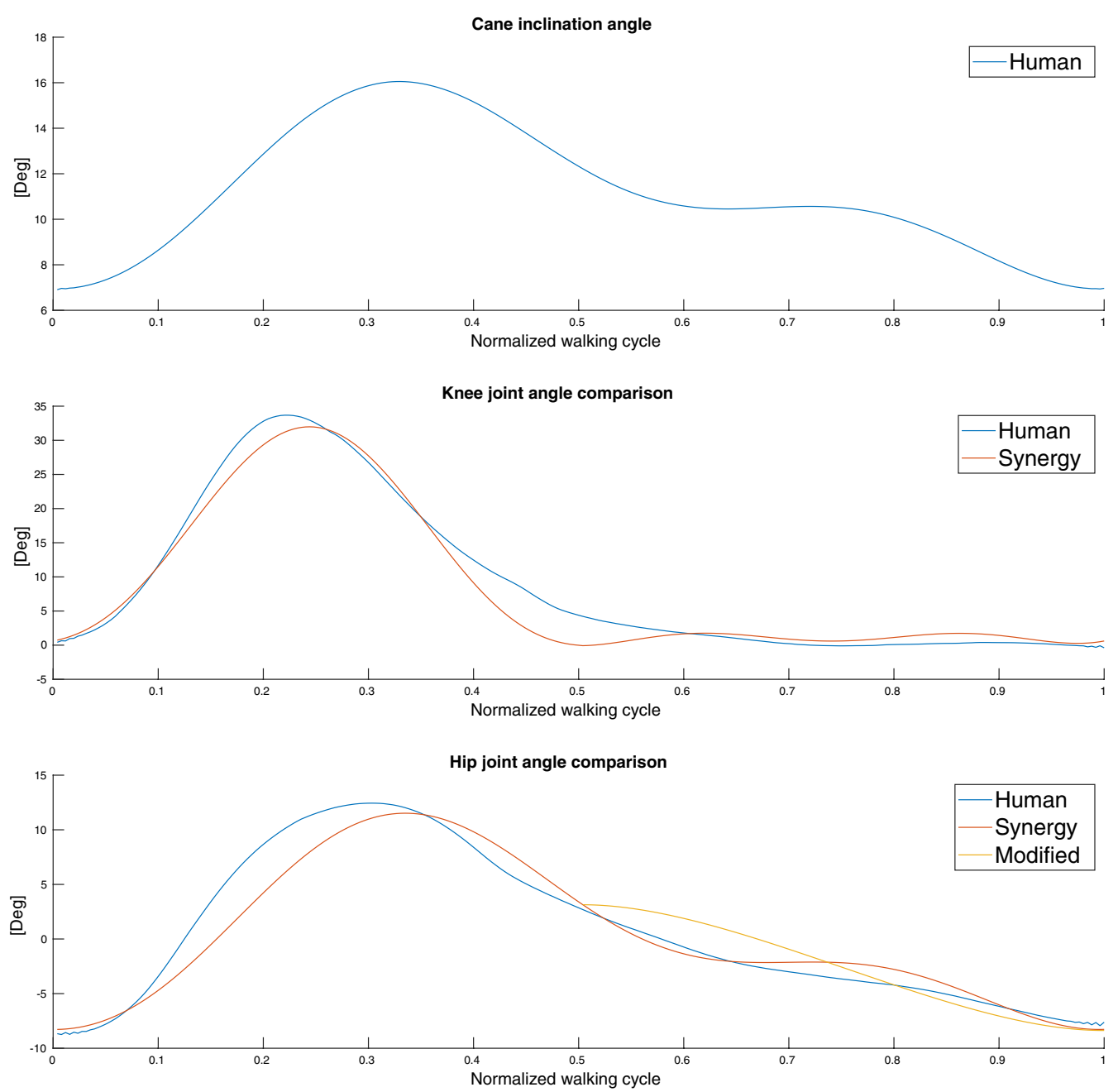

Fig. 9 Comparison of human gait, gait generated suing synergy and modified gait

leading the robot to fall within six steps. It can be seen from these experimental results that gait modification greatly improved the walking stability.

Figure 9 shows the hip and knee joint angles during one walking cycle. From top to bottom is the inclination angle of the cane, the knee joint angle, and the hip joint angle. The blue line represents the joint trajectory extracted from human walking with a cane. The red line represents the joint trajectory generated by human walking synergy. The yellow line represents the joint trajectory generated by human walking synergy and modified using ZMP. Either the joint trajectory generated by human walking synergy or the joint trajectory modified using ZMP was close to the joint trajectory extracted from human walking with a cane.

\section{Discussion}

There are three important points for assistive control of lower-limb exoskeleton to assist a paraplegic patient during walking: (1) synchronization of exoskeleton's motion and user walking intention, (2) human-like gait generation and (3) walking stability.

The use of human walking synergy to generate gait can solve the problems of synchronization between user intention and exoskeleton motion as well as human-like gait. Firstly, the leg motion generated by human walking synergy was completely synchronized with the motion of the cane. The motion of the cane was coupled with the motion of lower limbs, and it is controlled by the user and therefore reacts with the intention of the user. Secondly, the leg motion generated by human walking synergy is similar to the human walking motion. The walking motion generated in this way is natural and compatible. 
The use of ZMP to modify the walking motion solves the stability problem. Firstly, the walking trajectory generated using ZMP becomes stable. Theoretically, The ZMP stays inside the support polygon thereby enabling the exoskeleton to keep stable during walking. Secondly, the robot is also involved in maintaining balance. In this case, the patients no longer rely solely on canes to maintain balance, as a consequence reduce the burden on patients.

The use of NIP ensures that the modified walking trajectory is similar to the human walking trajectory. For rapid generation and easy calculation, a combination of a linear inverted pendulum (LIP) and ZMP is often used to generate the walking trajectory in robot gait planning. However, the walking motion generated using LIP has a constant height of CoM, which is inconsistent with the walking characteristics of humans. The use of NIP makes up for this shortcoming, thereby enables the modified trajectory to conform to human walking habits.

The traditional lower extremity exoskeleton uses a preplanned walking trajectory. Its walking can be controlled in terms of when to start and stop, and the walking speed. The walking with pre-planned trajectory is continuous. However in our method, it is necessary to predict the landing point and walking cycle of each step before lifting the foot, the walking action generated by the proposed method in is step-by-step. This step-by-step walking may make paraplegic patient feel safe to use the exoskeleton at the early stage of rehabilitation, but for patients who are familiar with the exoskeleton, walking might feel unnatural. Therefore, the continuity of walking should be improved to better conform to the walking habits of human. In addition, the ability to control the robot through human motion synergy still needs to be investigated with real exoskeleton and patient.

\section{Conclusion}

This paper proposes an algorithm via the modification of gait generated based on walking synergy to estimate motion intention and control powered lower-limb exoskeleton. This proposed system treats the cane as an interface between the user and the exoskeleton. The cane motion was used to generate the walking motion using walking synergy. The walking synergy was extracted from the movement trajectory of a healthy subject walking with a pair of canes. This synergy synchronizes the walking motion and the intention of user. The zero moment point (ZMP) was used to modify the walking motion to enhance the walking stability. To enable the modified walking motion in imitating the human walking habits, the nonlinear inverted pendulum model for trajectory planning was applied. Instead of a real patient wearing an exoskeleton, a cane robot was used for the Gazebo simulation. The experimental result shows that the gait modification significantly improved the walking stability, and the use of nonlinear inverted pendulum model resulted to a joint trajectory that is similar to the joint trajectory of human walking.

\section{Authors' contributions}

$M L$ performed the experiments, analyzed the data and wrote the manuscript. TA and $\mathrm{YH}$ guided the research and experiments, and reviewed this manuscript. All authors read and approved the final manuscript.

\section{Funding}

This work was supported by JSPS KAKENHI $17 \mathrm{H} 03205$.

Availability of data and materials

Not applicable.

\section{Competing interests}

The authors declare that they have no competing interests.

Received: 24 January 2020 Accepted: 24 April 2020

Published online: 30 April 2020

\section{References}

1. Zoss AB, Kazerooni H, Chu A (2006) Biomechanical design of the berkeley lower extremity exoskeleton (bleex). IEEE/ASME Transact Mechatron 11(2):128-138

2. Walsh CJ, Endo K, Herr H (2007) A quasi-passive leg exoskeleton for loadcarrying augmentation. Int J Hum Robotics 4(03):487-506

3. Pratt JE, Krupp BT, Morse CJ, Collins SH (2004) The roboknee: an exoskeleton for enhancing strength and endurance during walking. Proceeding of IEEE International Conference on Robotics and Automation 3:2430-2435

4. Esquenazi A, Talaty M, Packel A, Saulino M (2012) The rewalk powered exoskeleton to restore ambulatory function to individuals with thoracic-level motor-complete spinal cord injury. Am J Phys Med Rehabil 91(11):911-921

5. Kilicarslan A, Prasad S, Grossman RG, Contreras-Vidal JL (2013) High accuracy decoding of user intentions using eeg to control a lower-body exoskeleton. In: Proceeding of IEEE International Conference on Engineering in Medicine and Biology Society, p 5606-5609

6. Veneman JF, Kruidhof R, Hekman EE, Ekkelenkamp R, Van Asseldonk EH, Van Der Kooij H (2007) Design and evaluation of the lopes exoskeleton robot for interactive gait rehabilitation. IEEE Transact Neural Syst Rehabil Eng 15(3):379-386

7. Tsukahara A, Kawanishi R, Hasegawa Y, Sankai Y (2010) Sit-to-stand and stand-to-sit transfer support for complete paraplegic patients with robot suit hal. Advanced robotics 24(11):1615-1638

8. Tsukahara A, Hasegawa Y, Sankai Y (2011) Gait support for complete spinal cord injury patient by synchronized leg-swing with hal. In: Proceeding of IEEE International Conference on Intelligent Robots and Systems, p $1737-1742$

9. Tsukahara A, Hasegawa Y, Eguchi K, Sankai Y (2015) Restoration of gait for spinal cord injury patients using hal with intention estimator for preferable swing speed. IEEE Transact Neural Syst Rehabil Eng 23(2):308-318

10. Suzuki K, Mito G, Kawamoto H, Hasegawa Y, Sankai Y (2007) Intentionbased walking support for paraplegia patients with robot suit hal. Adv Robotics 21(12):1441-1469

11. Strickland E (2012) Good-bye, wheelchair. IEEE Spectr 49(1):30-2

12. Ford MP, Wagenaar RC, Newell KM (2007) Arm constraint and walking in healthy adults. Gait Posture 26(1):135-141

13. Wannier T, Bastiaanse C, Colombo G, Dietz V (2001) Arm to leg coordination in humans during walking, creeping and swimming activities. Exp Brain Res 141(3):375-379

14. Balter JE, Zehr EP (2007) Neural coupling between the arms and legs during rhythmic locomotor-like cycling movement. J Neurophysiol 97(2):1809-1818 
15. Dietz V, Fouad K, Bastiaanse C (2001) Neuronal coordination of arm and leg movements during human locomotion. Eur J Neurosci 14(11):1906-1914

16. Dietz V (2011) Quadrupedal coordination of bipedal gait: implications for movement disorders. J Neurol 258(8):1406

17. Daffertshofer A, Lamoth CJ, Meijer OG, Beek PJ (2004) Pca in studying coordination and variability: a tutorial. Clin Biomech 19(4):415-428

18. Todorov E, Ghahramani Z (2004) Analysis of the synergies underlying complex hand manipulation. In: The 26th Annual International Conference of the IEEE Engineering in Medicine and Biology Society, IEEE. 2: p 4637-4640

19. Crocher V, Sahbani A, Robertson J, Roby-Brami A, Morel G (2012) Constraining upper limb synergies of hemiparetic patients using a robotic exoskeleton in the perspective of neuro-rehabilitation. IEEE Transact Neural Syst Rehabil Eng 20(3):247-257

20. Crocher $V$, Jarrassé N, Sahbani A, Roby-Brami A, Morel G (2011) Changing human upper-limb synergies with an exoskeleton using viscous fields. In: 2011 IEEE International Conference on Robotics and Automation, IEEE. p 4657-4663

21. Liu K, Xiong C (2013) A novel 10-dof exoskeleton rehabilitation robot based on the postural synergies of upper extremity movements. In: International Conference on Intelligent Robotics and Applications, Springer. p 363-372

22. Hassan M, Kadone H, Suzuki K, Sankai Y (2012) Exoskeleton robot control based on cane and body joint synergies. In: Proceeding of IEEE International Conference on Intelligent Robots and Systems, p 1609-1614

23. Hassan M, Kadone H, Suzuki K, Sankai Y (2014) Wearable gait measurement system with an instrumented cane for exoskeleton control. Sensors 14(1):1705-1722

24. Hassan M, Kadone H, Ueno T, Suzuki K, Sankai Y (2015) Feasibility study of wearable robot control based on upper and lower limbs synergies. In:
Proceeding of International Symposium on Micro-NanoMechatronics and Human Science, p 1-6

25. Vallery H, Van Asseldonk EH, Buss M, Van Der Kooij H (2008) Reference trajectory generation for rehabilitation robots: complementary limb motion estimation. IEEE Transact Neural Syst Rehabil Eng 17(1):23-30

26. Vallery H, Buss M (2006) Complementary limb motion estimation based on interjoint coordination using principal components analysis. In: Proceeding of IEEE Conference on computer aided control system design, Proceeding of IEEE International Conference on Control Applications, Proceeding of IEEE International Symposium on Intelligent Control, p 933-938

27. Sardain P, Bessonnet G (2004) Forces acting on a biped robot center of pressure-zero moment point. IEEE Transact Syst Man Cybern-Part A: Syst Hum. 34(5):630-637

28. Kajita S, Kanehiro F, Kaneko K, Fujiwara K, Harada K, Yokoi K Hirukawa: Biped walking pattern generation by using preview control of zeromoment point

29. Park JH, Kim KD (1998) Biped robot walking using gravity-compensated inverted pendulum mode and computed torque control. Proceedings of IEEE International Conference on Robotics and Automation 4:3528-3533

30. Sugihara T, Nakamura Y, Inoue H (2002) Real-time humanoid motion generation through zmp manipulation based on inverted pendulum control. Proceedings of IEEE International Conference on Robotics and Automation 2:1404-1409

\section{Publisher's Note}

Springer Nature remains neutral with regard to jurisdictional claims in published maps and institutional affiliations.

\section{Submit your manuscript to a SpringerOpen ${ }^{\odot}$ journal and benefit from:}

- Convenient online submission

- Rigorous peer review

- Open access: articles freely available online

- High visibility within the field

- Retaining the copyright to your article

Submit your next manuscript at $\boldsymbol{\nabla}$ springeropen.com 